\title{
An Adaptive Control for the Path Tracking of an Active Leg Prosthesis
}

\author{
Rahma Boucetta ${ }^{\mathrm{a}, 1, *}$, Wiem Abdallah ${ }^{\mathrm{b}, 2}$, Saloua Bel Hadj Ali ${ }^{\mathrm{c}, 3}$ \\ a Department of Physics, Sciences Faculty of Sfax, University of Sfax, Road of Soukra, Tunisia \\ ${ }^{b}$ MACS Lab. LR16ES22, ENIG, University of Gabes, Tunisia \\ ${ }^{\text {c }}$ Preparatory Institute for Engineering Studies-El Manar, Farhat Hached Campus, Tunis, Tunisia \\ ${ }^{1}$ rahma.boucetta@macs.tn, ${ }^{2}$ wiemabdallah87@yahoo.fr, ${ }^{3}$ saloua.belhadjali@enit.rnu.tn \\ * Corresponding Author
}

\section{ARTICLE INFO}

\section{Article History}

Received 24 May 2021

Revised 03 June 2021

Accepted 05 June 2021

\section{Keywords}

Active lower limb prosthesis;

Dynamics;

Ground forces;

TS fuzzy system;

PID control;

LS method

\section{ABSTRACT}

This paper presents an adaptive fuzzy-PID control strategy applied to an active lower limb prosthesis for trajectories tracking in normal walking, stairs climbing, and stairs descent. Trying to imitate a natural human limb, the prosthesis design challenges rehabilitating amputees to resume normal activities. A dynamic model of an ankle-knee active prosthesis is developed without ground reaction in a first case and introduces ground effect in a second one to ameliorate prothesis performances. The obtained models are used to synthesize a control strategy based on TS fuzzy concepts and PID control to reproduce human lower limb behavior in a normal gait and climb and descent of stairs. The RSME errors are calculated to evaluate and compare the various results performances and eventually show the capacity of the proposed control with ground reaction impact on trajectory tracking. The RMSE values obtained for the four outputs of the fuzzy controller are very small for the different modes of locomotion. Moreover, they become weaker when the ground reaction forces are added to the model to show the role of these forces for the body equilibrium maintaining during the gait cycle. The developed approach ensured good trajectories tracking compared to a healthy leg even in presence of disturbances.

This is an open access article under the CC-BY-SA license.

\section{Introduction}

Limb amputation is a common medical problem that makes millions of people suffering from worldwide. People undergoes amputation for several reasons as trauma from work accidents or road damage or war victims [1]. Moreover, a great percentage suffers from amputation due to complications resulting from dysvascular diseases associated with diabetes [2]. Among all physiological and psychological approaches to help amputees to rehabilitate, limb prostheses are essential to allow amputees resuming their daily activities.

Thousands of lower limb amputations registered worldwide cause loss of mobility of the person, what affect life quality to become unable to continue normally daily activities. After amputation, the most goal is the rapid return of the amputee to its daily activities as soon as possible. After cure, a lower limb prosthesis remains an essential assistive device to recover some missing locomotion functions [3]. 
The development of prosthetic devices answers to one of principal challenges for aging societies: The loss of mobility and physical capacity in general. If workers are encouraged to perform physical work until old age while preventing musculoskeletal troubles, or if the elderly try to stay mobile later in their life and avoid a sedentary lifestyle, or if a better rehabilitation of motor abilities after neurological damage can be obtained, each effect constitute a large socioeconomic impact based on societal inclusion, general health and population productivity [4].

The assumption of biorobotics discipline requires new approaches to permit adequate treatment and better easiness of motion for persons doing hard physical labor or facing loss of their motor skills. Considering the interaction human-devices, their design and their adoption present major challenge, related to control, safety and features [5].

A lot of adequate prostheses are designed to restore missing mobility for lower limb amputees as walking, running or going upstairs. The development of a new generation of lower limb prostheses with improved performances is not only extremely desired by amputees, but evenly constitute a promising product, particularly required by the world market.

Over the last decades, prosthetics industry had known a significant revolution due to development of material, electronics, sensors and actuators. Currently, available prostheses are belong to three different families: passive, semi-active and actives prostheses.

Passive prostheses are mechanical devices built of springs and dampers without any external power, and with the appearance of amputated limb, they can give some basic features such as support, stabilize, push or pull... accomplished habitually with other natural articulations and extremities [6]. The SACH foot was a wooden prosthesis covered on the outside with rubber [7], it had no flexion and didn't adapt to any type of ground. The ESAR prosthetic foot was a modern evolution of the SACH model, it was more flexible to give natural movement for the ankle joint [8]. Later, soft feet had been developed based on windlass: chain of connectors turned parallel to the mediolateral direction can be used as a foot link. Their major inconvenient was their incapacity to generate mechanical power and their inefficiency to adapt to changing needs of the amputee and absence of sensory feedback of limb states to the user.

The second model is the semi-active prosthesis that utilizes passive mechanical components controlled by active adjustable valves. The Raize and Elan prostheses used hydraulic concepts and their resistance can be adjusted manually, furthermore, stability was considerably improved on slopes and slippery surfaces. Both mechanisms should allow better adaptation to the ground [9]. Particularly, Elan used a sensor feedback as input for an adaptive control depending of gait slope. The most popular semi-active knee prothesis C-Leg established in 1997 had different sensors to identify the gait cycle and transmit data to the microprocessor that can control the damping of the hydraulic joint [9].

The third type of prostheses is the active prosthetic articulations that use integrated actuators in electric drive to manipulate directly kinematics and kinetics of the prosthetic joint. The metabolic power of walking is reduced and the patient velocity is increased compared to passive and semi-active prostheses [10]. Proprio is the first active prosthesis that can lift the foot during oscillation phase to avoid falls. It consists of different sensors that detect the actual state stabilizing linear actuator to the appropriate position [11]. IP (Intelligent Prosthesis) is constructed of pneumatic cylinder to ensure the angular movement of the knee joint during swing phase [12]. BiOM Power Foot created by iWalk company presents another concept destined to ankle joints [13]. The elastic function of the Achilles tendon is combined to a motor for the calf muscles behavior.

An active motor of knee joint is provided to the Power Knee [14]. The knee extension during stairs climbing, slopes or standing position is supported by a motor. SPARKy presents a project funded by the US Army to develop a new generation of prostheses with tendon actuator to minimize significantly the power need of electrical motors while providing to the amputee an 
improved movement of the ankle and pushing power [15].

[16] designed an autonomous active prosthesis destined to improve the mobility of transfemoral amputees to provide walking as a normal gait. [17] developed an active knee-ankle prosthesis based on an actuator with variable stiffness and energy transfer from knee to ankle.

To control mechanisms, modelisation by physical laws or empirical approaches is a primordial step to understand anatomical limbs and human locomotion to determine a mathematical model that can facilitate the system comprehension, and make it analyzable and controllable. Many simple and complex models were proposed to design lower limb prostheses. Among used models, inverse pendulum [18], compass [19], ballistic gait [20], passive and active dynamic models [21], [22], [23] are the most known models.

The main goal of this work is to develop a control strategy considering physical interaction between the amputee, the prosthesis and the environment. An adaptive TS fuzzy-PID controller is proposed to generate the adequate torques in terms of healthy limb data and external ground reaction effect. Section 2 presents the dynamic model of an active lower limb prosthesis in the cases of absence and presence of ground forces. The TS fuzzy control is developed in section 3 in order to provide healthy limb behavior to the active prosthesis in three kinds of locomotion: walking, climbing and descent of stairs. Section 4 shows a comparative assessment of simulation results between study cases concerning the guarantee of closed loop and trajectories tracking performances.

\section{Dynamics of the Prosthesis}

\subsection{Anatomy}

The human lower limb (Fig. 1) is composed of foot, leg, thigh and gluteal region. The articulations considered in the lower limb are hip, knee and ankle joints. Their main functions are mostly weight support and movement aptitude. To perform efficiently these functions, the lower limb is constructed of ridiculous bones of hip, femur and tibia. Moreover, articulations are built of bones joints to ensure motions and give mechanical support. These articulations, improved by muscles, can be performed furthermore by pivot or ball joints to achieve some movements such as flexion, extension, abduction or adduction.

\subsection{Human Gait Cycle}

Human walking is considered to place one foot (leg) forward followed by the second, and replace the same foot to repeat the cycle of movement. Therefore, walking is a cyclic activity and one cycle can be studied to understand the whole period of walking. To represent the walking cycle, it is necessary to divide it into two main phases : stance phase where foot is in contact with ground and swing phase during which the foot is free.

The gait cycle can be divided into 7 subphases, Fig. 2:

- Loading response (0 to 10\%): After the initial contact, the body weight transfers to the front limb and continues until the other limb is lifted to allow the swing.

- Mid-stance (10 to 30\%): The weight is aligned with the fore foot.

- Terminal stance (30 to 50\%): The second foot hits the ground to achieve single support period.

- Pre-swing (50 to 60\%): While sudden transfer of the body weight relieves rapidly the limb, the rear end contributes to a progression of preparation to the swing.

- Toe-off (60 to 73\%): The lifting off the ground trains the swing foot to be opposite to the stance one. 


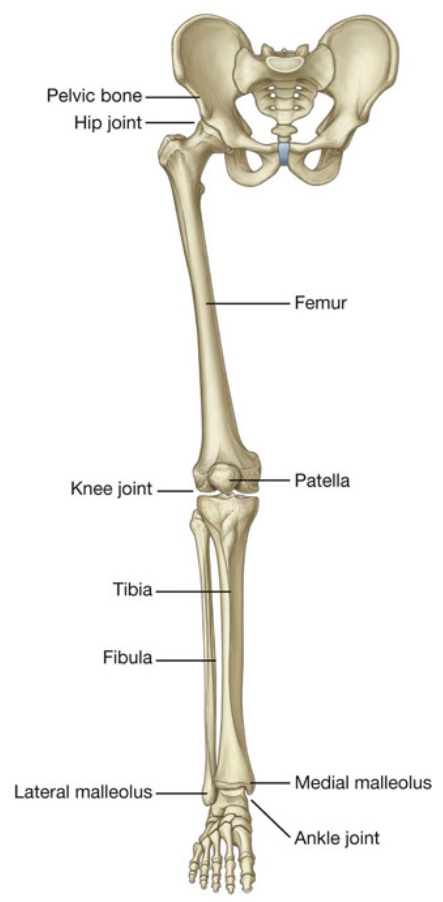

Fig 1. Lower limb skeleton

- Mid-swing (73 to 87\%): The swing foot is in front of the right limb to put the swing limb forward and the tibia vertical.

- Terminal swing (87 to 100\%): In the end, the foot hits the ground.

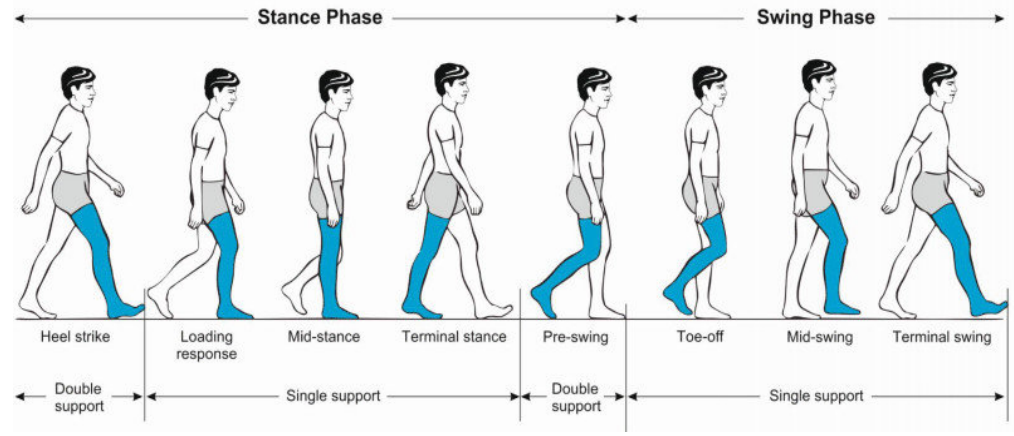

Fig 2. Different phases of a normal gait cycle

\subsection{Stairs Climb and Descent}

During climbing and descent of stairs, the lower limb moves cyclically like walking, and cycles of climb and descent are also subdivided to two phases: stance and swing periods. Each of these periods is characterized by a distant time between swing and stance phases.

\subsection{Ground Forces}

Most of human movements depend on body interaction with the ground. If a human executes activities as standing, walking, running or jumping, the body exerts continuously vertical and horizontal forces on the ground. In response, ground applies evenly equal and opposite forces to the body. These forces allow equilibrium of the body while performing all types of motor 
activities, and they called ground reaction, considered as the most important forces exerted on the body.

\subsection{Dynamic Model}

The human body is considered as a multi-body system composed of articulated rigid arms. Therefore, the Euler-Lagrange formalism is applied to generate the motion equations of the prosthetic leg during a normal gait cycle. The inverse dynamics are used to calculate torques and forces acting on knee and ankle links.

Basically, some assumptions should be considered: each segment has a mass assimilated to a mass center point with a fixed position, and constant moments of inertia and lengthes during movement, articulations are supposed pivot links. The lower limb can be assumed as a serial manipulator with rigid arms. The biomechanical model of the leg is represented in the sagittal plane. $L_{1}$ and $L_{2}$ indicate the segment lengthes. $r_{1}$ and $r_{2}$ designate the mass center positions. $\tau_{1}$ and $\tau_{2}$ are respectively the torques of ankle and knee joints. $m_{1}, I_{1}, m_{2}$ and $I_{2}$ are the masses and moments of inertia of leg and thigh segments respectively. The overall system has three variables $x, \theta_{1}$ and $\theta_{2}$ representing respectively foot distance, ankle and knee angles, Fig. 3.

The equations of movement can be obtained using the following form:

$$
\frac{d}{d t}\left(\frac{\partial L}{\partial \dot{q}}\right)-\frac{\partial L}{\partial q}=\Gamma, \quad q^{T}=\left[\theta_{1}, \theta_{2}, x\right]
$$

$\Gamma$ represents the generalized forces and torques vector obtained by the virtual work theorem with the following form:

$$
\Gamma=\left(\begin{array}{c}
\frac{\partial W}{\partial \theta_{1}} \\
\frac{\partial W}{\partial \theta_{2}} \\
\frac{\partial W}{\partial x}
\end{array}\right)=\left(\begin{array}{c}
\tau_{1}-\tau_{2} \\
\tau_{2} \\
0
\end{array}\right)
$$

The three equations of movement of the lower limb prosthesis can be expressed as:

$$
\begin{aligned}
& \tau_{1}=\left(m_{1} r_{1}^{2}+I_{1}+m_{2} L_{1}^{2}\right) \ddot{\theta}_{1}+\left(m_{2} r_{2}^{2}+I_{2}\right) \ddot{\theta}_{2}-\left(m_{1} r_{1}+m_{2} L_{1}\right) \ddot{x} \sin \theta_{1}-m_{2} r_{2} \ddot{x} \sin \theta_{2} \\
& +m_{2} r_{2} L_{1}\left(\ddot{\theta}_{1}+\ddot{\theta}_{2}\right) \cos \left(\theta_{2}-\theta_{1}\right)+m_{2} r_{2} L_{1}\left(\dot{\theta}_{2}^{2}-\dot{\theta}_{1}^{2}\right) \sin \left(\theta_{2}-\theta_{1}\right) \\
& +m_{1} r_{1} g \cos \theta_{1}+m_{2} L_{1} g \cos \theta_{1}+m_{2} r_{2} g \cos \theta_{2} \\
& \tau_{2}=\left(m_{2} r_{2}^{2}+I_{2}\right) \ddot{\theta}_{2}-m_{2} r_{2} \ddot{x} \sin \theta_{2}+m_{2} r_{2} L_{1} \ddot{\theta}_{1} \cos \left(\theta_{2}-\theta_{1}\right)+m_{2} r_{2} L_{1} \dot{\theta}_{1}^{2} \sin \left(\theta_{2}-\theta_{1}\right) \\
& +m_{2} r_{2} g \cos \theta_{2} \\
& 0=\left(m_{1}+m_{2}\right) \ddot{x}-\left(m_{1} r_{1}+m_{2} L_{1}\right)\left(\ddot{\theta}_{1} \sin \theta_{1}+\dot{\theta}_{1}^{2} \cos \theta_{1}\right)-m_{2} r_{2}\left(\ddot{\theta}_{2} \sin \theta_{2}+\dot{\theta}_{2}^{2} \cos \theta_{2}\right)
\end{aligned}
$$

The consideration of ground forces with horizontal and vertical components can modify the vector of the generalized forces as:

$$
\Gamma=\left(\begin{array}{c}
\tau_{1}-\tau_{2} \\
\tau_{2} \\
0
\end{array}\right)+J^{T}\left(\begin{array}{c}
F_{1} \\
F_{2}
\end{array}\right)=\left(\begin{array}{c}
\tau_{1}-\tau_{2}-F_{1} L_{1} \sin \theta_{1}+F_{2} L_{1} \cos \theta_{1} \\
\tau_{2}-F_{1} L_{2} \sin \theta_{2}+F_{2} L_{2} \cos \theta_{2} \\
F_{1}
\end{array}\right)
$$

$J$ is the Jacobian matrix. The highly coupled and nonlinear dynamic model of the active prosthesis has the following equations:

$$
\begin{aligned}
& \tau_{1}=\left(m_{1} r_{1}^{2}+I_{1}+m_{2} L_{1}^{2}\right) \ddot{\theta}_{1}+\left(m_{2} r_{2}^{2}+I_{2}\right) \ddot{\theta}_{2}-\left(m_{1} r_{1}+m_{2} L_{1}\right) \ddot{x} \sin \theta_{1}-m_{2} r_{2} \ddot{x} \sin \theta_{2} \\
& +m_{2} r_{2} L_{1}\left(\ddot{\theta}_{1}+\ddot{\theta}_{2}\right) \cos \left(\theta_{2}-\theta_{1}\right)+m_{2} r_{2} L_{1}\left(\dot{\theta}_{2}^{2}-\dot{\theta}_{1}^{2}\right) \sin \left(\theta_{2}-\theta_{1}\right)+m_{1} r_{1} g \cos \theta_{1} \\
& +m_{2} L_{1} g \cos \theta_{1}+m_{2} r_{2} g \cos \theta_{2}+F_{2} L_{1} \sin \theta_{1}+F_{2} L_{2} \sin \theta_{2}-F_{1} L_{1} \cos \theta_{1}-F_{1} L_{2} \cos \theta_{2}
\end{aligned}
$$




$$
\begin{aligned}
& \tau_{2}=\left(m_{2} r_{2}^{2}+I_{2}\right) \ddot{\theta}_{2}-m_{2} r_{2} \ddot{x} \sin \theta_{2}+m_{2} r_{2} L_{1} \ddot{\theta}_{1} \cos \left(\theta_{2}-\theta_{1}\right)+m_{2} r_{2} L_{1} \dot{\theta}_{1}^{2} \sin \left(\theta_{2}-\theta_{1}\right) \\
& \quad+m_{2} r_{2} g \cos \theta_{2}-F_{1} L_{2} \cos \theta_{2}+F_{2} L_{2} \sin \theta_{2} \\
& F_{1}=\left(m_{1}+m_{2}\right) \ddot{x}-\left(m_{1} r_{1}+m_{2} L_{1}\right)\left(\ddot{\theta}_{1} \sin \theta_{1}+\dot{\theta}_{1}^{2} \cos \theta_{1}\right)-m_{2} r_{2}\left(\ddot{\theta}_{2} \sin \theta_{2}+\dot{\theta}_{2}^{2} \cos \theta_{2}\right)
\end{aligned}
$$

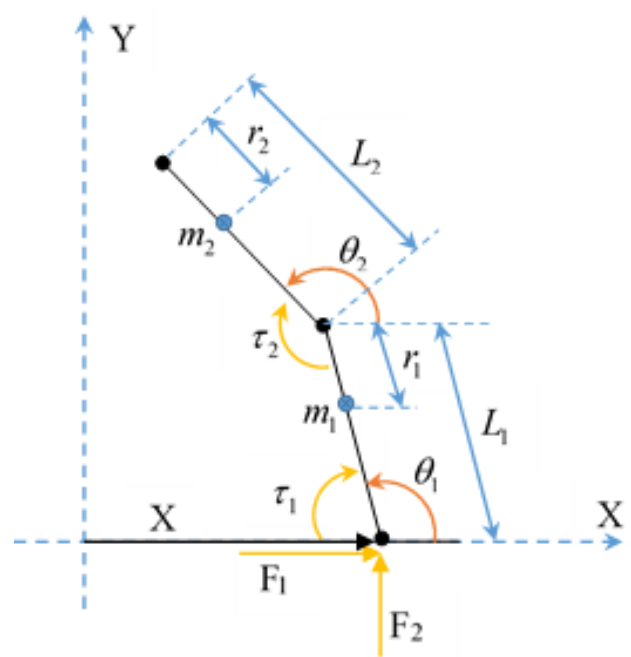

Fig 3. Active prosthesis model

\section{Adaptive Fuzzy Controller}

\subsection{Without Ground Reaction}

The prosthetic leg should be controlled by torques generated from a fuzzy controller and must be follow trajectories of displacement similar to those performed by a healthy leg in the three forms of locomotion. The tibia and femur angles present the inputs of the inference mechanism, the deduced outputs give torques and desired articular angles of the prosthesis. Depending on femur and tibia angles, the FIS (Fuzzy Inference System) decides the right mode to execute in terms of real data from the natural leg, Fig. 4.

Two PID controllers are used as secondary controllers to correct prosthetic position errors for the knee and ankle articulations compared to desired values generated from external disturbances. Based on real data base of a healthy limb, the fuzzy controller must provide the mode and the phase of the gait cycle in which the prosthesis is located, and generate nominal torques $\tau_{d_{K}}$ and $\tau_{d_{A}}$ and the reference trajectories $\theta_{d_{K}}$ and $\theta_{d_{A}}$ for the knee and ankle articulations.

\subsubsection{Membership Functions}

The training data of the controller are partitioned into subsets extracted from subphases of healthy leg activities and characterized each one by a membership function. If each sample variable is assumed normally and independently distributed, the distribution of the sample mean is supposed to be normal (Gaussian).

If the size of each sample is $N$, for $X_{1}, X_{2}, . ., X_{n}$ of independent variables, the mean and the variance are given by:

$$
\begin{gathered}
E(\bar{X})=m=\frac{\bar{X}_{1}+\bar{X}_{2}+\ldots+\bar{X}_{n}}{N} \\
V(\bar{X})=\frac{\sigma_{1}^{2}+\sigma_{2}^{2}+\ldots+\sigma_{n}^{2}}{N^{2}}
\end{gathered}
$$




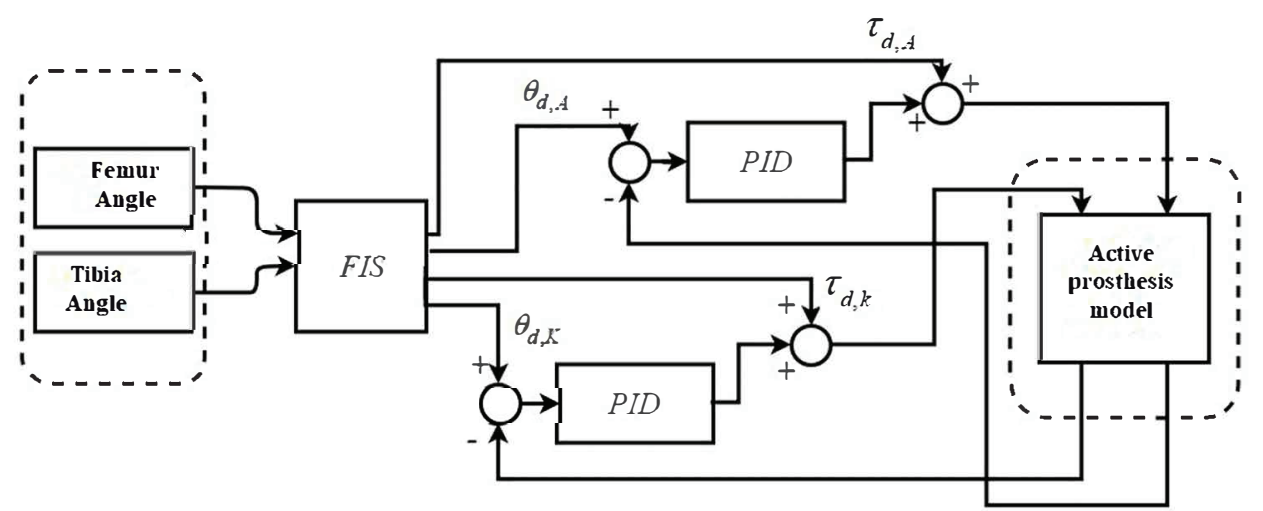

Fig 4. Control structure of the active prosthesis

where $\sigma_{i}(i=1, . ., n)$ designates the standard error of the distribution expressed as:

$$
\sigma=\frac{1}{N} \sqrt{\sigma_{1}^{2}+\sigma_{2}^{2}+\ldots+\sigma_{n}^{2}}
$$

\subsubsection{Rule Base}

The TSK model is adopted by the inference mechanism used for the controller. For two inputs, with 7 fuzzy sets each one, the maximum number of rules must not exceed 49 rules. However, during the gait cycle, the thigh and the leg should remain in the same phase anytime. The obtained If - Then rules are summarized in a relational matrix illustrated by Fig. 5.

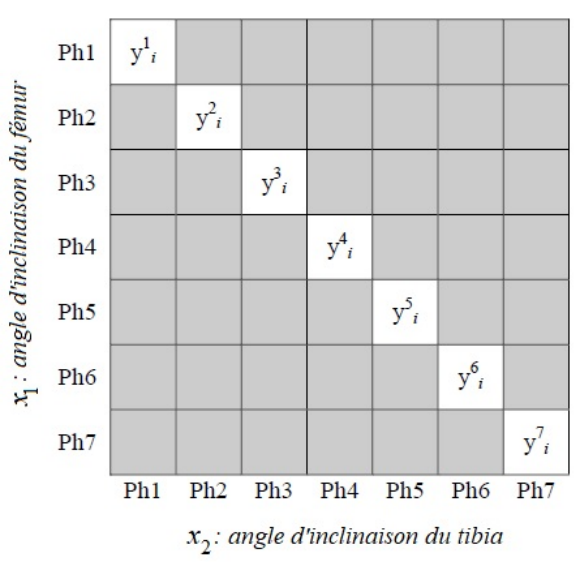

Fig 5. Rule base of the gait cycle

The variable $y_{i}^{\ell}$ corresponds to the $i^{\text {th }}$ output of the $\ell^{\text {th }}$ fuzzy rule. The rule base of the controller without ground reaction is given by the following expression:

$$
R_{\ell} \text { : If } x_{1} \text { is } P h_{1}^{\ell} \text { and } x_{2} \text { is } P h_{2}^{\ell} \text { Then }\left\{\begin{array}{l}
y_{1}^{\ell}(x)=c_{1,0}^{\ell}+c_{1,1}^{\ell} x_{1}+c_{1,2}^{\ell} x_{2} \\
y_{2}^{\ell}(x)=c_{2,0}^{\ell}+c_{2,1}^{\ell} x_{1}+c_{2,2}^{\ell} x_{2} \\
y_{3}^{\ell}(x)=c_{3,0}^{\ell}+c_{3,1}^{\ell} x_{1}+c_{3,2}^{\ell} x_{2} \\
y_{4}^{\ell}(x)=c_{4,0}^{\ell}+c_{4,1}^{\ell} x_{1}+c_{4,2}^{\ell} x_{2}
\end{array}\right.
$$

where $x_{1}$ and $x_{2}$ are the two inputs of the controller and $y_{1}^{\ell}(x), . ., y_{4}^{\ell}(x)$ the outputs for the $\ell^{\text {th }}$ rule designating respectively the knee and ankle angles. 
The $i^{\text {th }}$ crisp output of the FIS can be obtained using the following equation:

$$
y_{i, \ell, T S K}(x)=\frac{\sum_{\ell=1}^{M} f^{\ell}(x)\left(c_{i, 0}^{\ell}+c_{i, 1}^{\ell} x_{1}+c_{i, 2}^{\ell} x_{2}\right)}{\sum_{\ell=1}^{M} f^{\ell}(x)}
$$

where $M$ is the number of fuzzy rules, $f^{\ell}(x)$ represents the activation degree of the fuzzy rule defined as:

$$
f^{\ell}(x)=\mathrm{T}_{k=1}^{p} \mu_{F_{j}^{\ell}}\left(x_{j}\right)
$$

where $T$ is the t-norm generally chosen the minimum or the product, and $\mu_{F_{j}^{\ell}}\left(x_{j}\right)$ presents the membership degree of the $j^{\text {th }}$ input associated to the $\ell^{\text {th }}$ rule.

\subsubsection{Controller Parameters Optimization}

The LS (Least Square) method is applied to obtain estimated parameters according to training data. Considering $\bar{f}^{\ell}(x)$ as:

$$
\bar{f}^{\ell}(x)=\frac{f^{\ell}(x)}{\sum_{\ell=1}^{M} f^{\ell}(x)}
$$

The equation (10) can be written as:

$$
y_{i, \ell, T S K}(x)=\sum_{\ell=1}^{M} \bar{f}^{\ell}(x) y_{i}^{\ell}(x)=\sum_{\ell=1}^{M} \bar{f}^{\ell}(x)\left(c_{i, 0}^{\ell}+c_{i, 1}^{\ell} x_{1}+c_{i, 2}^{\ell} x_{2}\right)
$$

In matrix form:

$$
y_{i, \ell, T S K}(x)=\phi C_{i}^{T}
$$

where

$$
\begin{aligned}
C_{i} & =\left[\begin{array}{llll}
c_{i, 0}^{1} \ldots c_{i, 0}^{M} & c_{i, 1}^{1} \ldots c_{i, 1}^{M} & c_{i, 2}^{1} \ldots c_{i, 2}^{M}
\end{array}\right] \\
\phi & =\left[\begin{array}{llll}
\bar{f}^{1}(x) \ldots \bar{f}^{M}(x) & x_{1} \bar{f}^{1}(x) \ldots x_{1} \bar{f}^{M}(x) & x_{2} \bar{f}^{1}(x) \ldots x_{2} \bar{f}^{M}(x)
\end{array}\right]
\end{aligned}
$$

The size of $\phi$ matrix is the number of training pairs and may be expressed as:

$$
\phi=\left[\begin{array}{ccc}
\bar{f}_{1}^{1} \ldots \bar{f}_{M}^{1} & x_{1}^{1} \bar{f}_{1}^{1} \ldots x_{1}^{1} \bar{f}_{M}^{1} & x_{2}^{1} \bar{f}_{1}^{1} \ldots x_{2}^{1} \bar{f}_{M}^{1} \\
\bar{f}_{1}^{2} \ldots \bar{f}_{M}^{2} & x_{1}^{2} \bar{f}_{1}^{2} \ldots x_{1}^{2} \bar{f}_{M}^{2} & x_{2}^{2} \bar{f}_{1}^{2} \ldots x_{2}^{2} \bar{f}_{M}^{2} \\
\bar{f}_{1}^{N} \ldots \bar{f}_{M}^{N} & x_{1}^{N} \bar{f}_{1}^{N} \ldots x_{1}^{N} \bar{f}_{M}^{N} & x_{2}^{N} \bar{f}_{1}^{N} \ldots x_{2}^{N} \bar{f}_{M}^{N}
\end{array}\right]
$$

where $x_{j}^{n}$ is the $j^{\text {th }}$ input of the $n^{\text {th }}$ training pair. $C$, the parameters vector, can be obtained using the following formula:

$$
C_{i}=\left(\phi^{T} \phi\right)^{-1} \phi^{T} y_{i}
$$

\subsection{With Ground Reaction}

To study the ground forces impact on the active prosthesis, four inputs are introduced in the fuzzy controller: the tibia and femur angles, and the horizontal and vertical components of the ground reaction, Fig. 6. 


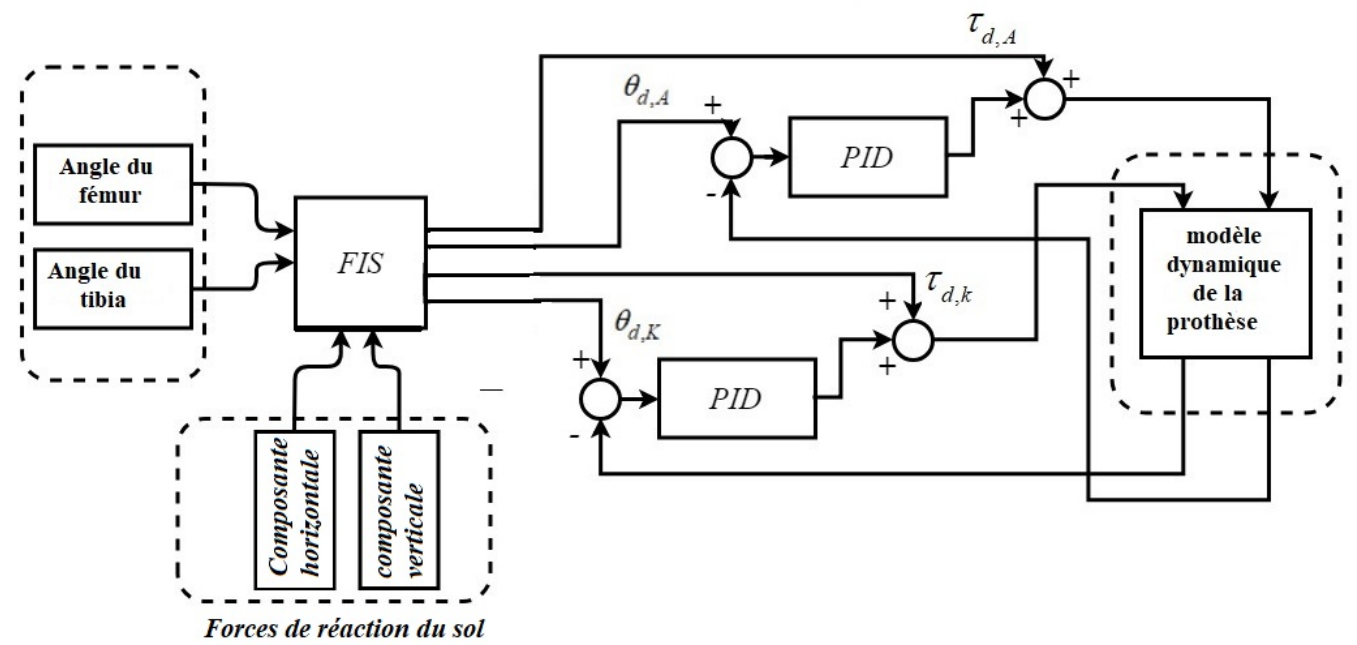

Fig 6. Control structure for the prosthesis with ground reaction

The general form of the fuzzy rule in this case has the following expression:

$$
\begin{aligned}
& R_{l}: \text { If } x_{1} \text { is } P h_{1}^{\ell} \text { and } x_{2} \text { is } P h_{2}^{\ell} \\
& \text { and } x_{3} \text { is } P h_{3}^{\ell} \text { and } x_{4} \text { is } P h_{4}^{\ell}
\end{aligned}
$$

Then

$$
\left\{\begin{array}{l}
y_{1}^{\ell}(x)=c_{1,0}^{\ell}+c_{1,1}^{\ell} x_{1}+c_{1,2}^{\ell} x_{2}+c_{1,3}^{\ell} x_{3}+c_{1,4}^{\ell} x_{4} \\
y_{2}^{\ell}(x)=c_{2,0}^{\ell}+c_{2,1}^{\ell} x_{1}+c_{2,2}^{\ell} x_{2}+c_{2,3}^{\ell} x_{3}+c_{2,4}^{\ell} x_{4} \\
y_{3}^{\ell}(x)=c_{3,0}^{\ell}+c_{3,1}^{\ell} x_{1}+c_{3,2}^{\ell} x_{2}+c_{3,3}^{\ell} x_{3}+c_{3,4}^{\ell} x_{4} \\
y_{4}^{\ell}(x)=c_{4,0}^{\ell}+c_{4,1}^{\ell} x_{1}+c_{4,2}^{\ell} x_{2}+c_{4,3}^{\ell} x_{3}+c_{4,4}^{\ell} x_{4}
\end{array}\right.
$$

\section{Simulation Results}

Simulation results can be obtained based on anthropomorphic data of a person with $1.75 \mathrm{~m}$ height and $75 \mathrm{Kg}$ weight. Table 1 gives the required parameters of control.

Table 1. Parameters of the prosthesis

\begin{tabular}{|c|c|c|}
\hline Parameter & Leg & Thigh \\
\hline Mass $(\mathrm{kg})$ & 7.5 & 3.488 \\
\hline Moment of inertia $\left(\mathrm{kg} / \mathrm{m}^{2} \mathbf{)}\right.$ & 0.144 & 0.059 \\
\hline Center of mass $(\mathrm{m})$ & 0.186 & 0.186 \\
\hline
\end{tabular}

The amount of 51 pairs of data are introduced in the fuzzy controller to evaluate the system performances. The outputs of the proposed controller generate the desired trajectories for the angles and torques of prosthetic articulations to mimic real performances of a healthy leg. The fuzzy controller parameters are obtained using the LS method of optimization. Two PID controllers with online adjusted parameters are added to correct prosthetic position error compared to desired values. The result is shown in Fig. 7 to Fig. 18.

Fig. 7 presents controller output for the gait cycle compared to the real leg. Fig. 7 (a)-(b) is knee angle and knee torque. Fig. 7(c)-(d) is ankle angle and ankle torque. It can be seen that the system controller can follow the real angle. 


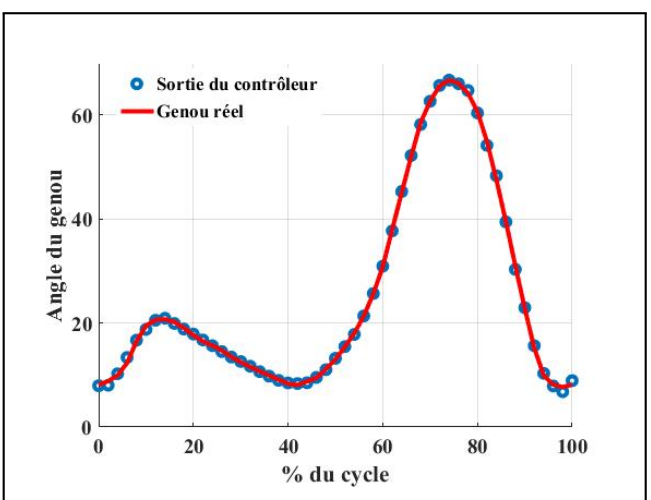

(a) Knee angle

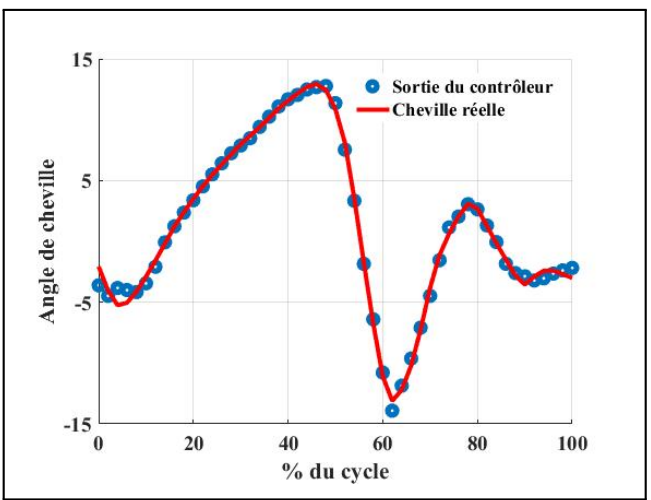

(c) Ankle angle

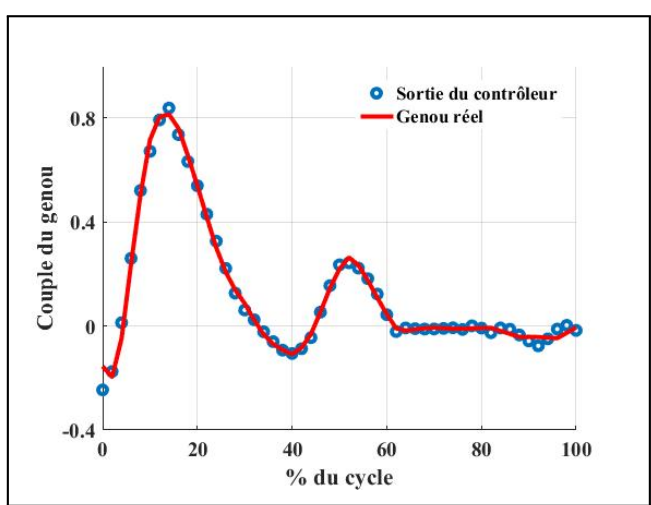

(b) Knee torque

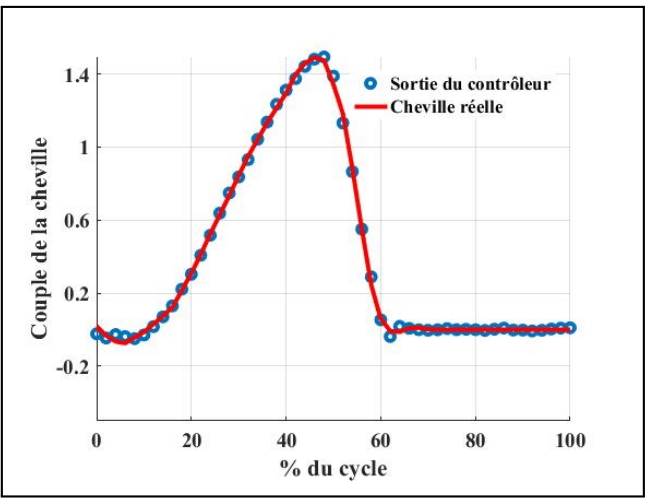

(d) Ankle torque

Fig 7. Controller outputs (blue) for a gait cycle compared to real leg (red)

Fig. 8 shows about Prosthetic angles evolution for a gait cycle compared to real leg in ankle and knee. It can be seen that the prosthetic angle can follow the real leg for the gain cycle.

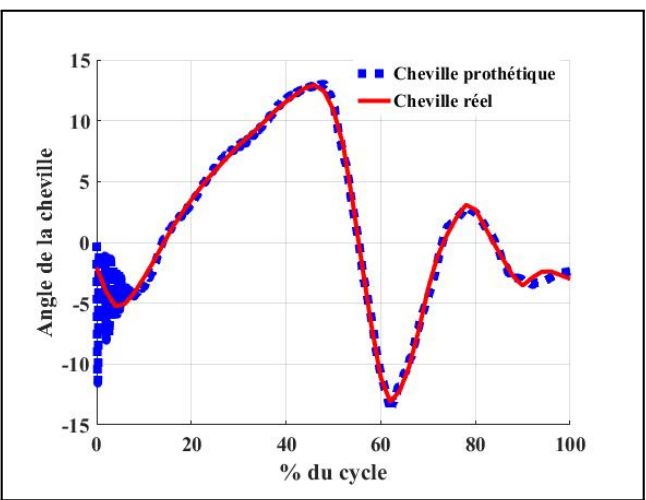

(a) Ankle

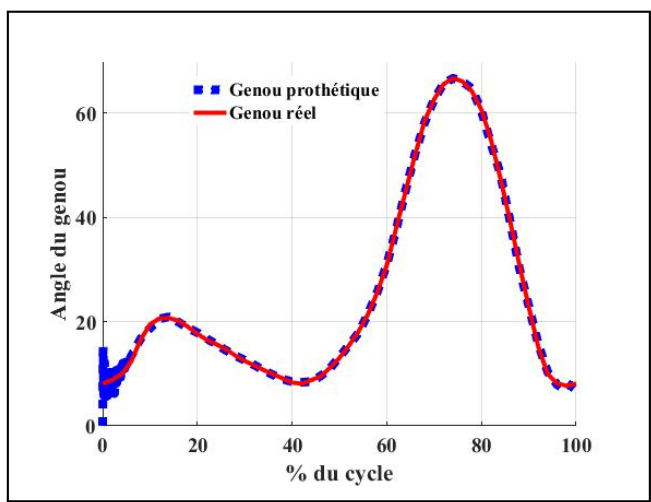

(b) Knee

Fig 8. Prosthetic angles evolution (blue) for a gait cycle compared to real leg (red)

Fig. 9 presents controller output for the stairs climb compared to the real leg. It can be seen that the system controller can follow the real angle. 


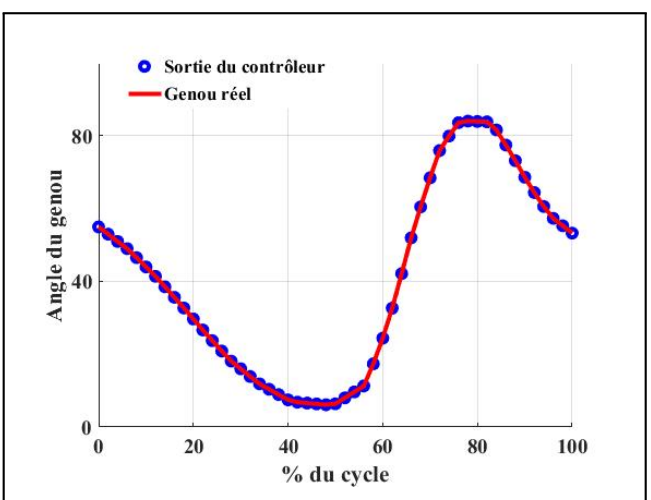

(a) Knee angle

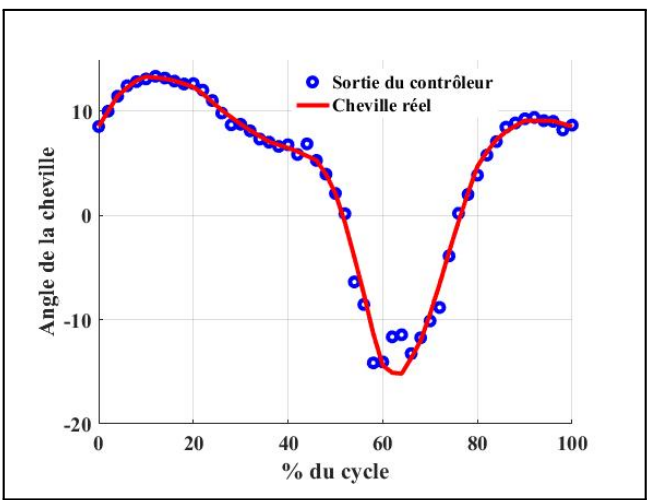

(c) Ankle angle

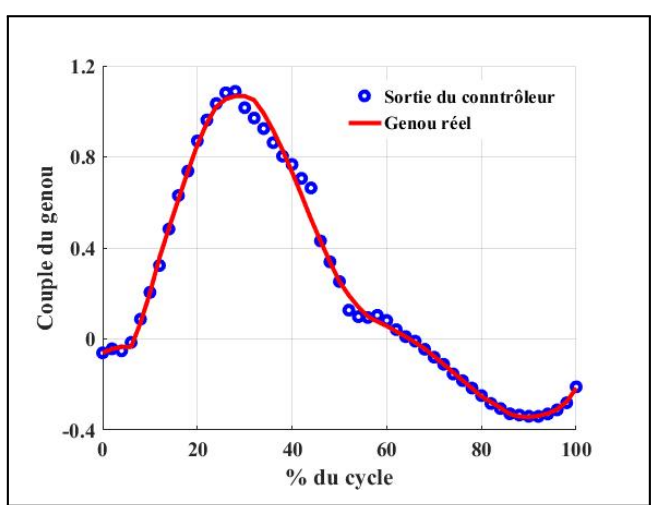

(b) Knee torque

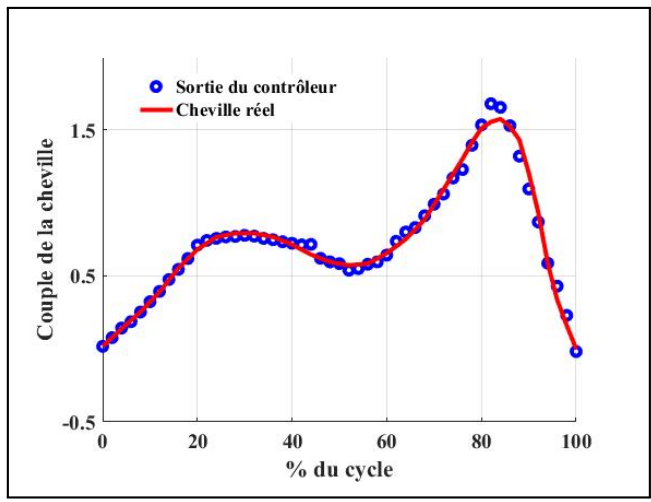

(d) Ankle torque

Fig 9. Controller outputs (blue) for stairs climb compared to real leg (red)

Fig. 10 shows about Prosthetic angles evolution for stairs climb compared to real leg in ankle and knee. It can be seen that the prosthetic angle can follow the real leg for stairs climb.

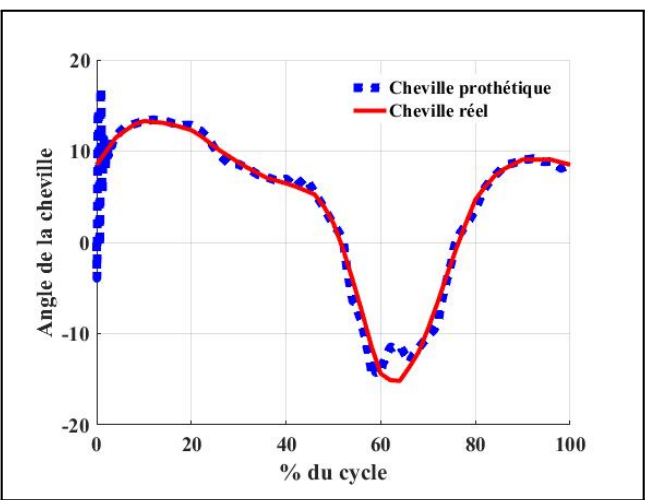

(a) Ankle

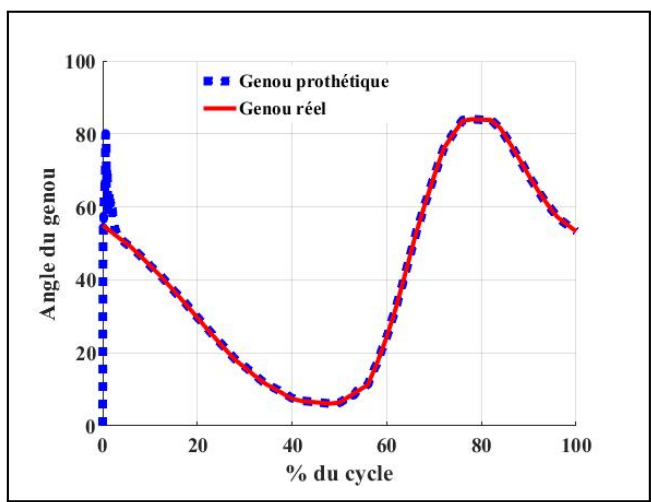

(b) Knee

Fig 10. Prosthetic angles evolution (blue) for stairs climb compared to real leg (red)

Fig. 11 presents controller output for the stairs descent compared to the real leg. It can be seen that the system controller can follow the real angle with small overshoot. 


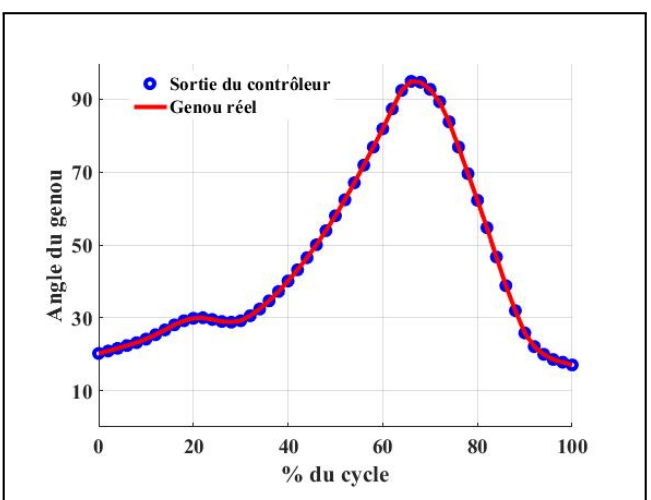

(a) Knee angle

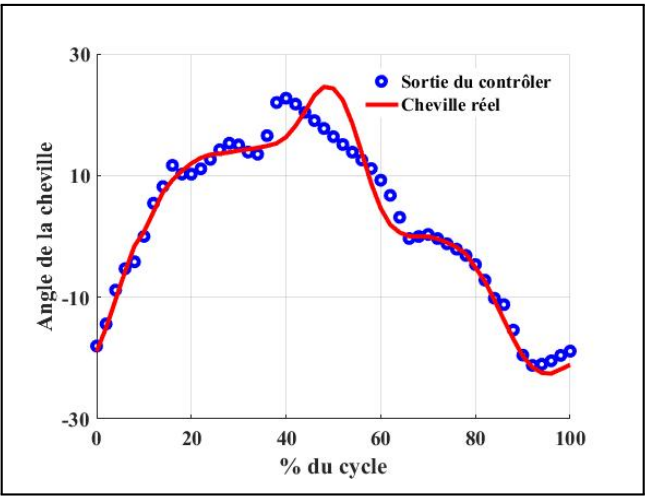

(c) Ankle angle

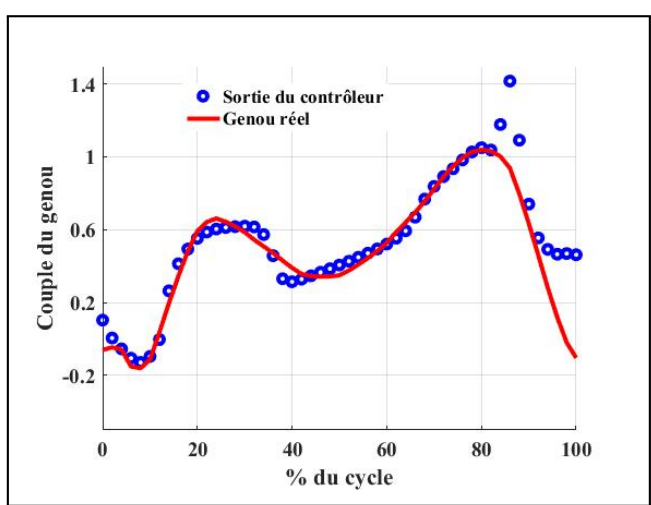

(b) Knee torque

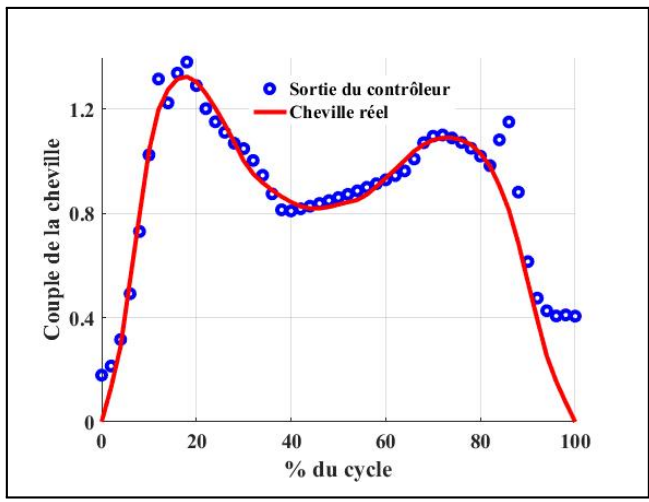

(d) Ankle torque

Fig 11. Controller outputs (blue) for stairs descent compared to real leg (red)

Fig. 12 shows about Prosthetic angles evolution for stairs descent compared to real leg in ankle and knee. It can be seen that the prosthetic angle can follow the real leg for stairs descent.

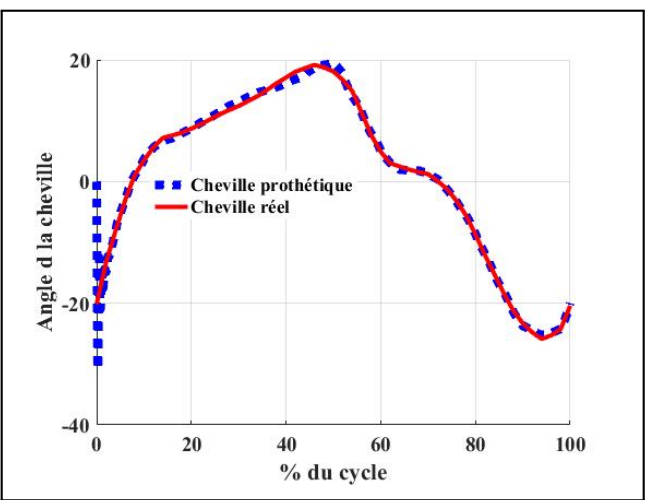

(a) Ankle

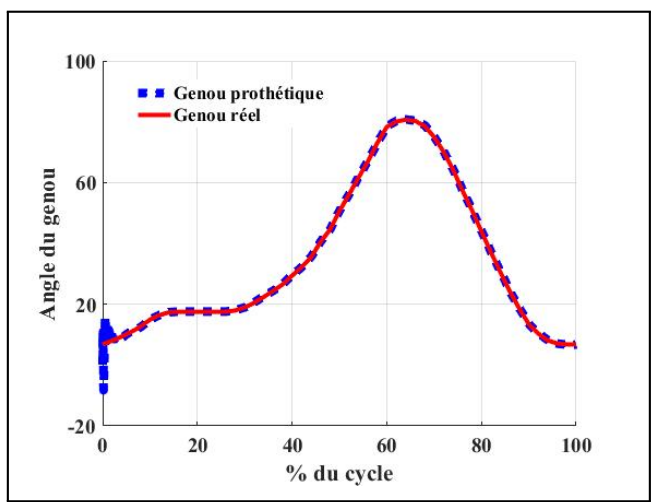

(b) Knee

Fig 12. Prosthetic angles evolution (blue) for stairs descent compared to real leg (red)

Fig. 13 presents controller output for a gait cycle with ground reaction compared to the real leg. It can be seen that the system controller can follow the real angle. 


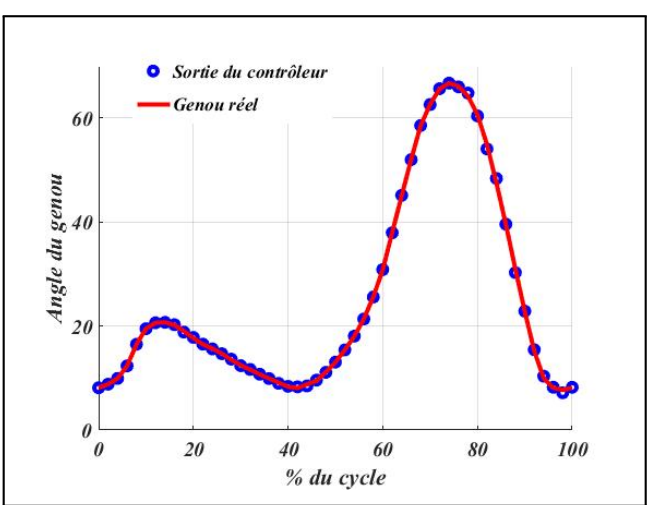

(a) Knee angle

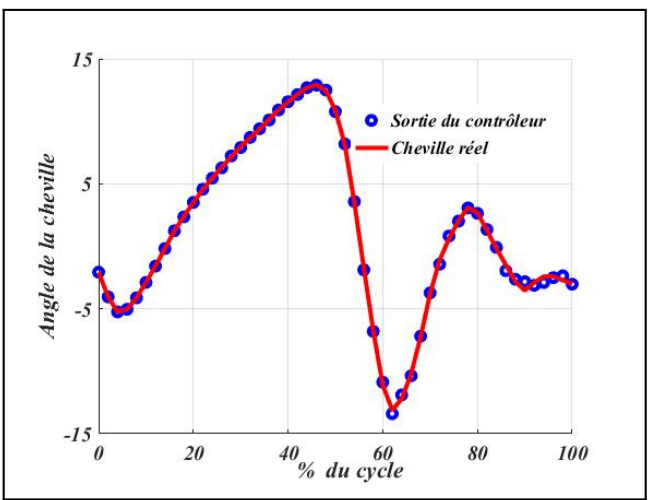

(c) Ankle angle

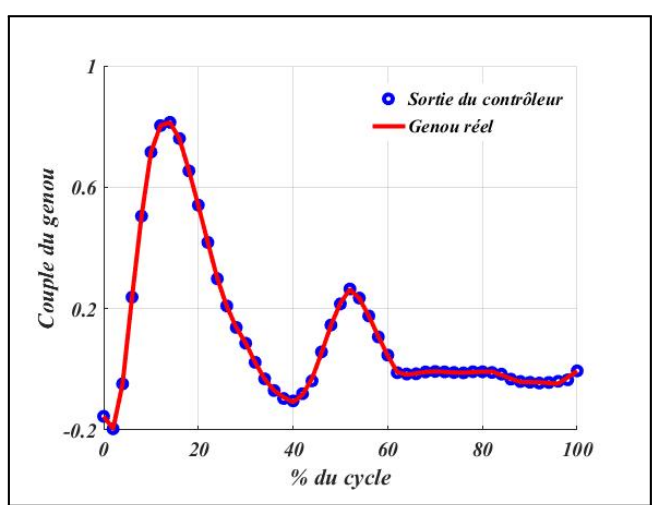

(b) Knee torque

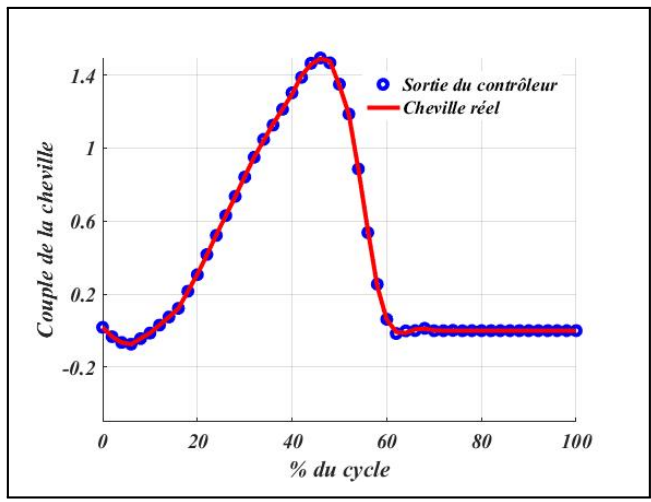

(d) Ankle torque

Fig 13. Controller outputs (blue) for a gait cycle with ground reaction compared to a real leg (red)

Fig. 14 shows about Prosthetic angles evolution for a gait cycle with ground reaction compared to real leg in ankle and knee. It can be seen that the prosthetic angle can follow the real leg for a gait cycle with ground reaction.

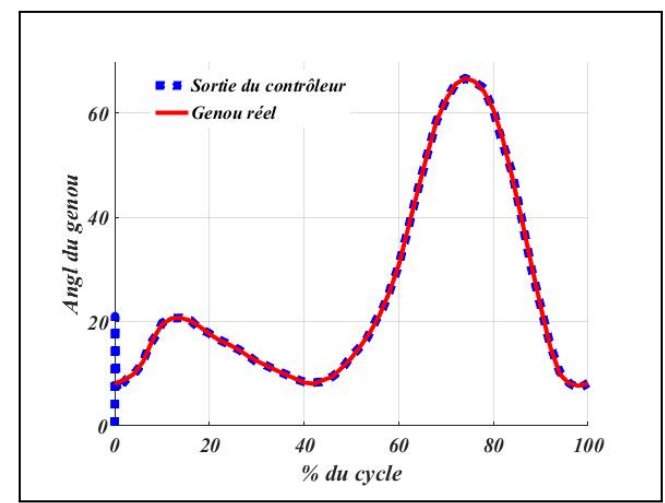

(a) Ankle

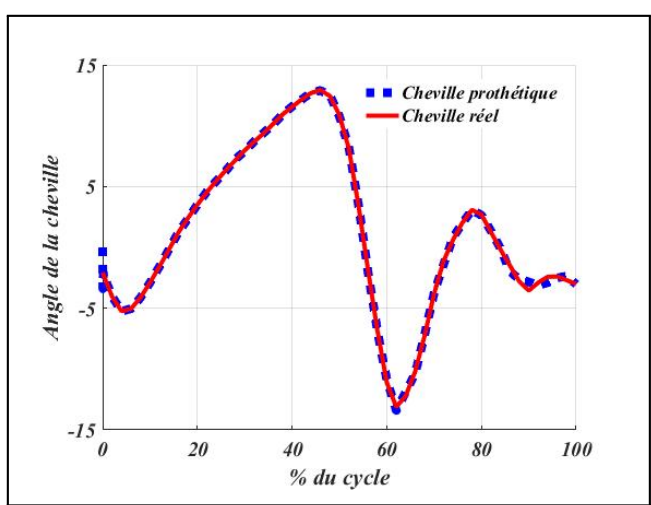

(b) Knee

Fig 14. Prosthetic angles evolution (blue) for a gait cycle with ground reaction compared to real leg (red)

Fig. 15 presents controller output for stairs climb with ground reaction compared to the real leg. It can be seen that the system controller can follow the real angle. 


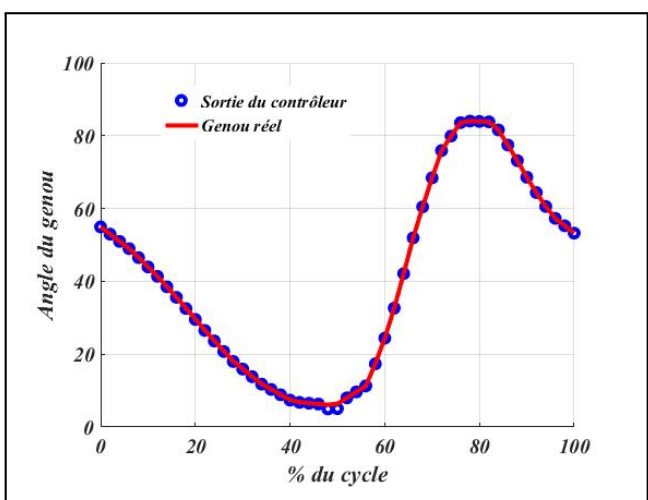

(a) Knee angle

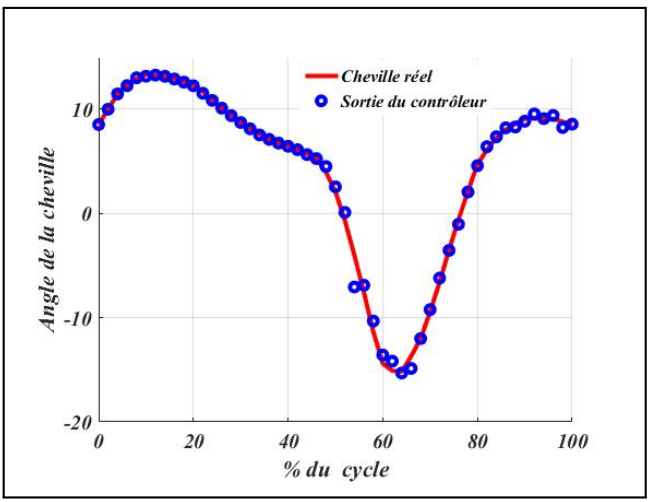

(c) Ankle angle

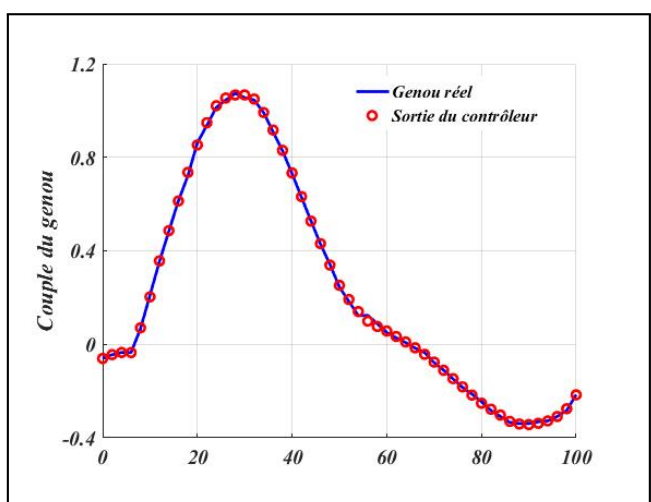

(b) Knee torque

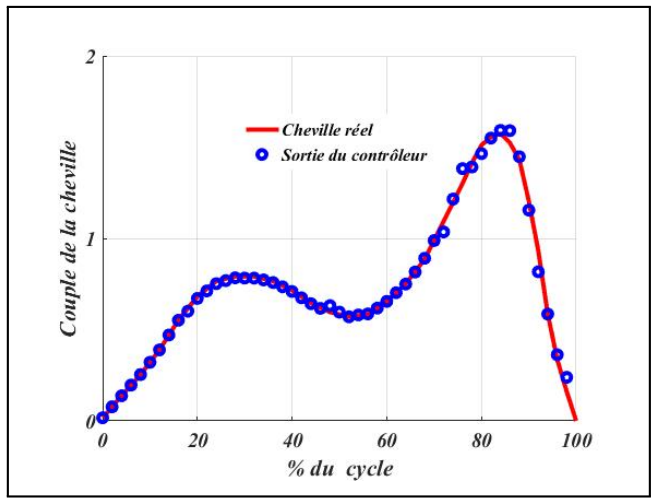

(d) Ankle torque

Fig 15. Controller outputs (blue) for stairs climb with ground reaction compared to real leg (red)

Fig. 16 shows about Prosthetic angles evolution for stairs climb with ground reaction compared to real leg in ankle and knee. It can be seen that the prosthetic angle can follow the real leg for stairs climb with ground reaction.

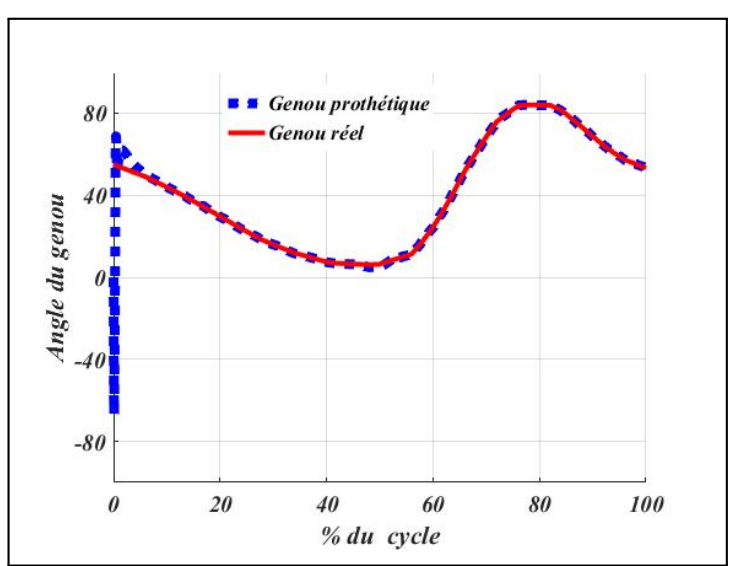

(a) Ankle

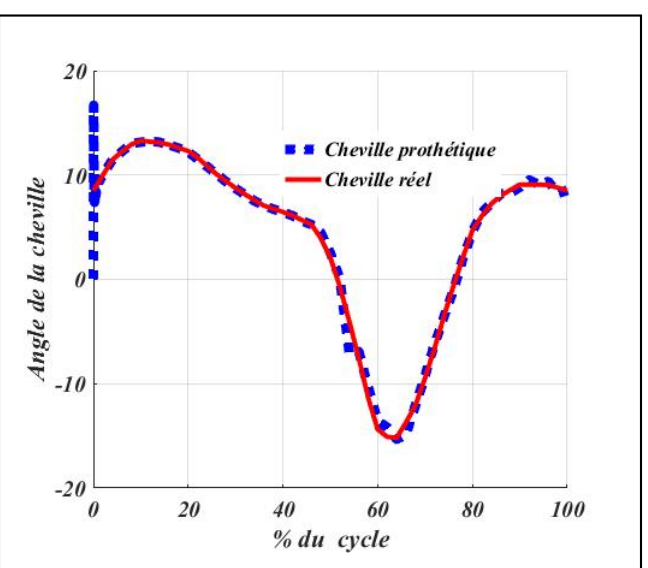

(b) Knee

Fig 16. Prosthetic angles evolution (blue) for stairs climb with ground reaction compared to real leg (red)

Fig. 17 presents controller output for stairs descent with ground reaction compared to the real leg. It can be seen that the system controller can follow the real angle. 


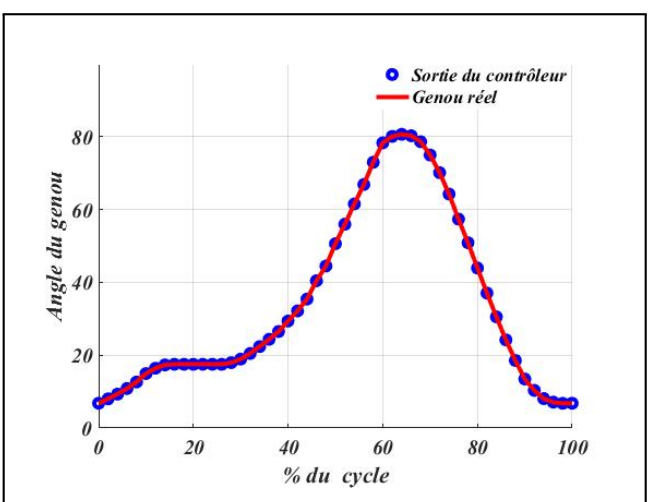

(a) Knee angle

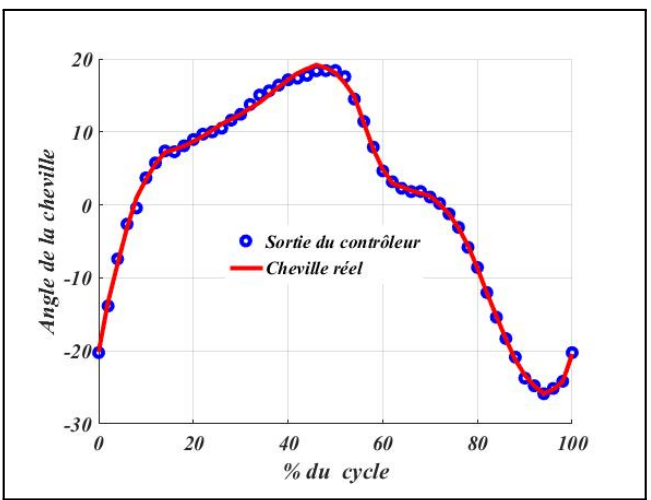

(c) Ankle angle

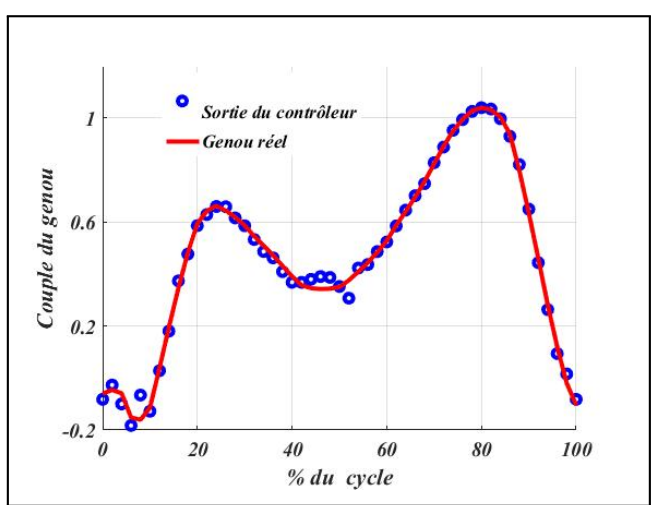

(b) Knee angle

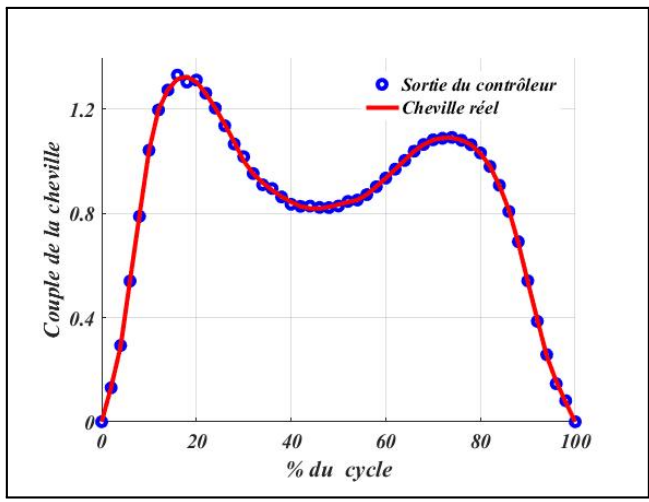

(d) Ankle torque

Fig 17. Controller outputs (blue) for stairs descent with ground reaction compared to real leg (red)

Fig. 18 shows about Prosthetic angles evolution for stairs descent with ground reaction compared to real leg in ankle and knee. It can be seen that the prosthetic angle can follow the real leg for stairs descent with ground reaction.

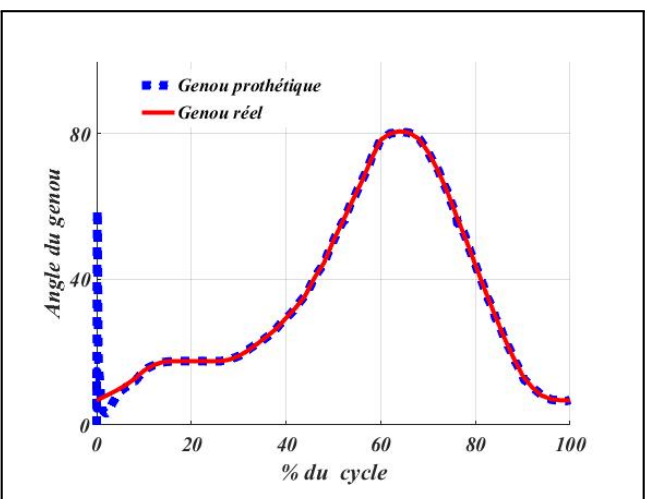

(a) Ankle

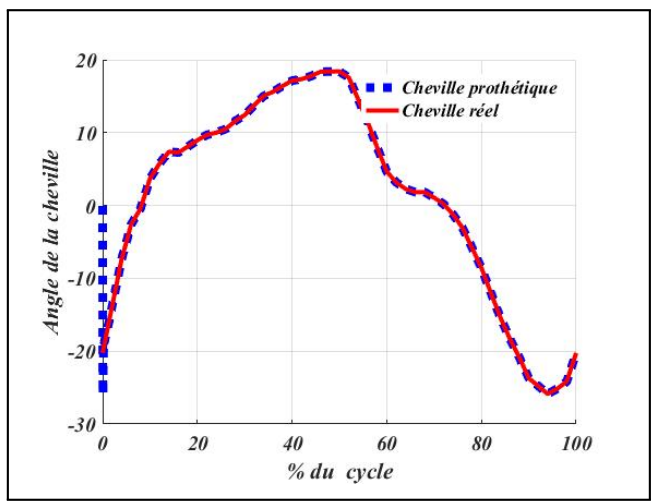

(b) Knee

Fig 18. Prosthetic angles evolution (blue) for stairs descent with ground reaction compared to real leg (red)

The comparative assessment is accomplished for the three modes of locomotion: walking (Figs. 7-8 \& Figs. 13-14), stairs climb (Figs. 9-10 \& Figs. 15-16) and descent (Figs. 11-12 \& Figs. 
17-18) without and with ground reaction. For each cycle, output responses present initially short oscillations affecting angular variation of joints when ground forces missed, whereas with ground reaction effect, an improvement can be observed, and start-up oscillations are properly attenuated to offer more stability for the active prothesis.

To evaluate inference system performance designed in prediction of corresponding values to data set, the RMSE (Root Mean Square Error) of training data obtained by LS method are calculated and compared for the two cases as:

$$
R M S E=\sqrt{\frac{1}{n} \sum_{1}^{n} e_{i}} \quad n=1, \ldots, 51
$$

Table 2 to Table 5 present the RMSE values for the four outputs of the controller in the three cases of locomotion.

Table 2. RMSE for the $1^{\text {th }}$ output of the controller

\begin{tabular}{|c|c|c|c|}
\hline$y_{1}$ & Walking & Stairs climb & Stairs descent \\
\hline Without ground reaction & 0.1255 & $1.5684 e^{-4}$ & $1.0333 e^{-6}$ \\
\hline With ground reaction & 0.0432 & $1.5063 e^{-5}$ & $2.6641 e^{-6}$ \\
\hline
\end{tabular}

Table 3. RMSE for the $2^{\text {th }}$ output of the controller

\begin{tabular}{|c|c|c|c|}
\hline$y_{2}$ & Walking & Stairs climb & Stairs descent \\
\hline Without ground reaction & 0.0 .146 & 0.0104 & 0.0350 \\
\hline With ground reaction & 0.0043 & 0.0033 & 0.0207 \\
\hline
\end{tabular}

Table 4. RMSE for the $3^{\text {th }}$ output of the controller

\begin{tabular}{|c|c|c|c|}
\hline$y_{3}$ & Walking & Stairs climb & Stairs descent \\
\hline Without ground reaction & 0.1269 & 0.0519 & 0.0796 \\
\hline With ground reaction & 0.0250 & 0.0287 & 0.0600 \\
\hline
\end{tabular}

Table 5. RMSE for the $4^{\text {th }}$ output of the controller

\begin{tabular}{|c|c|c|c|}
\hline$y_{4}$ & Walking & Stairs climb & Stairs descent \\
\hline Without ground reaction & 0.089 & 0.0188 & 0.0263 \\
\hline With ground reaction & $4.9494 e^{-4}$ & 0.0109 & 0.0031 \\
\hline
\end{tabular}

The RMSE values obtained for the four outputs of the fuzzy controller are very small for the different modes of locomotion in Table 2 - Table 5. Moreover, they become more weaker when the ground reaction forces are added to the model to show the role of these forces for the body equilibrium maintaining during the gait cycle.

\section{Conclusion}

In this paper, an adaptive TS-PID controller was proposed for the control of an active lower limb prosthesis. Real data collected from healthy leg are used to perform the controller parameters. To improve control performances, the ground reaction forces are introduced into the prosthesis model and consequently generated the adequate torques and desired angles for the prosthetic knee and ankle. The developed approach ensured good trajectories tracking compared to a healthy leg even in presence of disturbances. In a future work, a neuro-fuzzy controller will 
be designed and tested on a test bed to improve prosthetic performances in a large number of locomotion.

\section{Acknowledgement}

This work was supported by the Ministry of the Higher Education and Scientific Research in Tunisia.

\section{References}

[1] G. Bastas, "Lower Limb Amputations," Essentials of Physical Medicine and Rehabilitation, pp. 658-663, 2020. https://doi.org/10.1016/B978-0-323-54947-9.00120-6

[2] S. S. Mathew-Steiner, D. Khona, and C. K. Sen, "The diabetic foot," Wound Healing, Tissue Repair, and Regeneration in Diabetes, pp. 3-12, 2020. https://doi.org/10.1016/ B978-0-12-816413-6.00001-0

[3] A. S. Sarvestani, and A. T. Azam, "Amputation: A Ten-Year Survey," Journal of Trauma and Emergency Medicine, vol. 18, no. 3, pp. 126-128, 2013. https://www.ncbi.nlm.nih.gov/ pmc/articles/PMC3864397/

[4] M. Windrich, M. Grimmer, O. Christ, S. Rinderknecht, and P. Beckerle, "Active lower limb prosthetics: a systematic review of design issues and solutions," BioMedical Engineering OnLine, vol. 15, no. 3, p. 140, 2016. https://doi.org/10.1186/s12938-016-0284-9

[5] S. Thoualt, and A. Allais, "La biorobotique, Definition et objectifs," 2015. Retrieved from http://tpe-biorobotique.e-monsite.com/pages/biorobotique/la-biorobotique.html

[6] R. Seymour, Prosthetics and orthotics: lower limb and spinal, Lippincott Williams \& Wilkins, 2002. https://books.google.co.id/books?id=2KIqqHS5fVoC

[7] M. Grimmer, Powered lower limb prostheses, Technische Universität, 2015. http:// tuprints.ulb.tu-darmstadt.de/4382/

[8] A. K. Lapre, "Semi-Active Damping for an Intelligent Adaptive Ankle Prosthesis," Thesis, University of Massachusetts Amherst, 2012.https://scholarworks.umass.edu/theses/ 805/

[9] P. Artemiadis, Neuro-robotics: From brain machine interfaces to rehabilitation robotics, Springer, 2014. https://doi.org/10.1007/978-94-017-8932-5

[10] D. S. Pieringer, M. Grimmer, M. F. Russold, and R. Riener, "Review of the actuators of active knee prostheses and their target design outputs for activities of daily living," International Conference on Rehabilitation Robotics ICORR'2017, pp. 1246-1253, 2017. https://doi.org/ 10.1109/ICORR.2017.8009420

[11] B. J. Darter and J. M. Wilken. "Energetic consequences of using a prosthesis with adaptive ankle motion during slope walking in persons with a transtibial amputation," Prosthetics and Orthotics International, vol. 38, pp. 5-11, 2014. https://doi.org/10.1177/ 0309364613481489

[12] J. G. Buckley, W. D. Spence, and S. E. Solomonidis, "Energy cost of walking: Comparison of intelligent prosthesis with conventional mechanism," Archives of Physical Medicine and Rehabilitation, vol. 78, pp. 330-333, 1997. https://doi.org/10.1016/S0003-9993(97) 90044-7 
[13] V. Agrawal, R. S. Gailey, I. A. Gaunaurd, C. O'Toole, A. Finnieston, and R. Tolchin, "Comparison of four different categories of prosthetic feet during ramp ambulation in unilateral transtibial amputees," Prosthetics and Orthotics International, vol. 39, no. 5, pp. 380-389, 2015. https//doi.org/10.1016/S0003-9993(97)90044-7

[14] E. J. Wolf, V. Q. Everding, A. A. Linberg, J. M. Czerniecki, and C.J.M. Gambel, "Comparison of the Power Knee and C-Leg during step-up and sit-to-stand tasks," Gait and posture, vol. 38, no. 3, pp. 397-402, 2013. https://doi.org/10.1016/j.gaitpost.2013.01.007

[15] R. Bellman, M. A. Holgate, and T. Sugar, "Design of an active robotic ankle prosthesis with two actuated degrees of freedom using regenerative kinetics," 2nd IEEE RAS and EMBS International Conference on Biomedical Robotics and Biomechatronics BioRob, pp. 511516, 2008. https://doi.org/10.1109/BIOROB.2008.4762887

[16] F. C. SupIV, A. Bohara, and M. Goldfarb, "Design and Control of a Powered Transfemoral Prosthesis," The International Journal of Robotics Research, vol. 27, no. 2, pp. 263-273, 2008. https://doi.org/10.1177/0278364907084588

[17] J. Geeroms, L. L. Flynn, R. J. Fabian, and D. Lefeber, "Ankle-knee prosthesis with powered ankle and energy transfer for CYBERLEGs," IEEE International Conference on Rehabilitation Robotics, pp. 1-20, 2013. https://doi.org/10.1109/ICORR.2013.6650352

[18] P. Aerts, R.F. Ker, D. De Clercq, D.W. Ilsley, and R.McN. Alexander, "The mechanical properties of the human heel pad: A paradox resolved," Journal of Biomechanics, vol. 28, no. 11, pp. 1299-1308, 1995. https://doi.org/10.1016/0021-9290(95)00009-7

[19] J. R. Usherwood, K. L. Szymanek, and M.A. Daley, "Compass gait mechanics account for top walking speeds in ducks and humans," Journal of Experimental Biology, vol. 211, no. 23, pp. 3744-9, 2008. https://doi.org/10.1242/jeb.023416

[20] S. Mochon, and T. A. McMahon, "Ballistic walking: an improved model," Mathematical Biosciences, vol. 52, pp. 241-260, 1980. https://doi.org/10.1016/0025-5564(80)90070-X

[21] T. McGeer, "Passive Dynamic Walking," The International Journal of Robotics Research, vol. 9, no. 2, pp. 62-82, 1990. https://doi.org/10.1177/027836499000900206

[22] A. D. Kuo, "Energetics of Actively Powered Locomotion Using the Simplest Walking Model," Journal of Biomechanical Engineering, vol. 124, no. 1, pp. 113-20, 2002. https://doi.org/ $10.1115 / 1.1427703$

[23] M. Nobile, P. Perego, L. Piccinini, E. Mani, A. Rossi, M. Bellina, and M. Massimo, "Further evidence of complex motor dysfunction in drug naive children with autism using automatic motion analysis of gait," Autism, vol. 15, no. 3, pp. 263-83, 2011. https://doi.org/10.1177/ 1362361309356929 